\section{Aligned Growth of Stem Cells Derived from Ceria/PLGA Composite}

Tissue engineering continues to be a field of great interest due to its tremendous biomedical implications. In particular, hybrid organic-inorganic materials show great promise for tailoring biocompatibility, mechanical and electrical properties to a greater extent than the commonly used biopolymers alone. Cerium oxide $\left(\mathrm{CeO}_{2}\right)$, or ceria, is known for its ability to quench free radicals, and it acts as an antioxidant by converting $\mathrm{Ce}^{3+}$ to $\mathrm{Ce}^{4+}$ in the presence of oxygen radicals. It is known that free radical formation can hinder biological cell proliferation, which is a requisite for tissue engineering. In the May 25 issue of $A d$ vanced Functional Materials (DOI: 10.1002/ adfm.200902363; p. 1617), E. Traversa and C. Mandoli of the National Institute for Materials Science, Japan; F. Pagliari of the University of Rome Tor Vergata, Italy; and their colleagues report the aligned growth of murine-derived cardiac and mesenchymal adult stem cells on a ceria/PLGA (poly(D,L-lactic-co-glycolic acid) biopolymer) hybrid composite substrate. Mesenchymal stem cells are multipotent stem cells that have been shown to differentiate into bone cells, cartilage cells, and fat cells.

The researchers used patterned molds for solvent casting of the ceria/PLGA films. Ceria nanoparticles (5-8 nm grain size) were aligned along parallel lines, both straight and spirally grooved, to evaluate the orientation of the ceramic on cell growth. Nano-ceria with weight percent amounts of $0,5,10$, and 20 were used. Nanocomposites with randomly oriented ceria nanoparticles were also formed as a control. These hybrid composites were
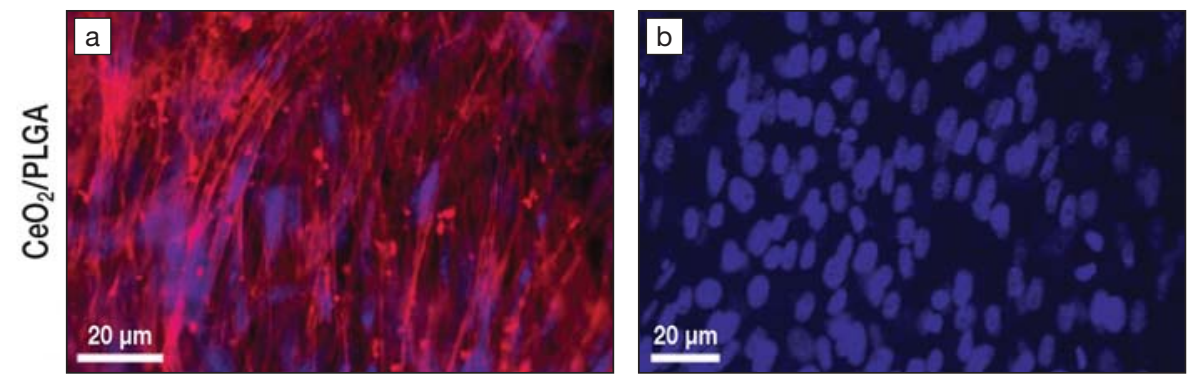

Figure 1. Murine-derived cardiac stem cell grafts produced after 6 days in vitro over spirally pre-patterned composites. The images were acquired after (a) F-actin (red) and (b) nuclei (blue) stain under immunofluorescence and refer to $10 \mathrm{wt} \% \mathrm{CeO}_{2} / \mathrm{PLGA}$ seeded composites. Reproduced with permission from Advanced Functional Materials 20 (10) (2010) 1617; DOI: 10.1002/adfm.200902363. (c) 2010 Wiley-VCH.

then examined for cell adhesion, growth, and proliferation using the stem cells.

After three days in vitro (DIV) culturing, coverage of the scaffold surfaces with healthy cells was observed, with the cells growing in an aligned fashion following the ceria nanoparticles pattern; no alignment was observed with a random distribution or in the absence of the ceria particles. This growth continued after six DIV as well. The largest cellular densities were observed for the $10 \mathrm{wt} \%$ ceria-loaded substrate, which also showed the greatest toughness in terms of mechanical properties (see Figure 1).

To understand whether the chemical nature of ceria or the physical/morphological changes yielded the excellent biological response observed, $6 \%$ titania $\left(\mathrm{TiO}_{2}\right)$ /PLGA nanocomposites formed on similarly patterned molds were also subjected to the same cultures for comparison. Titania shows less reactivity to reactive oxidant species than ceria. The chemical nature of the ceramic appeared not to be relevant for aligned cellular growth. However, the ceria composites yielded much better cellular response, in terms of attachment and cell density, likely because titania does not biochemically interact with cells as does ceria. The results showed that aligned cellular growth occurs along aligned ceramic particles within the composite ridges. The particles give topographic signals that cells sense and recognize.

According to the researchers, this work demonstrates that cells can be instructed to behave in a specific biological manner (aligned growth) simply by physical factors, rather than chemical factors. Conventionally, most biological processes are explained through chains of chemical reactions, whereas in the present case, the driving signal is physical. The results suggest that materials properties might directly control biological processes. This could represent a revolution in understanding biology through the lens of materials science.

GOPAL R. RAO

\section{Nanoscale Displacements Detected by Evanescent Optical Coupling from an Optical Fiber to a Si Cantilever}

Mechanical displacements at the nanoscale are often measured by optical interferometry that requires the use of a coherent light source. Using non-interferometric techniques, such as beam deflection, allows the ability to relax this constraint. Recently, O. Basarir, K.L. Ekinci, and their colleagues at Boston University accurately measured displacements of a silicon microcantilever due to its evanescent optical coupling to a weakly guided mode of a silica optical fiber taper placed in its vicinity.

As described in the June 1 issue of Optics Letters (DOI: 10.1364/OL.35.001792; p. 1792), light of a broadband-amplified spontaneous emission light source is shone through the optical fiber taper. The output optical power transmission (around $38 \mu \mathrm{W}$ ) was recorded on a photodetector. Optical coupling between the cantilever and the optical fiber arose when the evanescent wave tail of the weakly guided mode is partly lost into the higher index material of the Si cantilever.

The transmission of the fiber strongly depended on its distance from the cantilever, with a maximal sensitivity at a separation of $\sim 580 \mathrm{~nm}$, before the coupling completely disappeared at large distances between the cantilever and taper. Small thermal oscillations of the cantilever position caused the transmission to vary at the cantilever oscillation frequency. The researchers integrated the high-frequency spectrum of the thermomechanical noise and compared this to the transmission data in order to determine the sensitivity of their approach.

Thermomechanical oscillations of the cantilever at room temperature of the order of tens of picometers could be resolved with a resolution limited by the light source noise. This sensitive and simple technique does not require the use of a coherent light source and could be adapted to scanning probe microscopy or in nanoscale displacement detection.

ELSA COUDERC 